\title{
DESIGN OF A BIO BASED APPROACH FOR CONGESTION CONTROL IN WIRELESS SENSOR NETWORK
}

\author{
Suchitha.S \\ Research Scholar, Bharathiar University \\ Coimbatore, TamilNadu, India \\ Dr.B.G.Prasanthi \\ Associate Professor, Computer Science Department \\ St.Joseph's College, Bangalore, India
}

\begin{abstract}
In wireless sensor networks (WSNs), congestion can occur due to more data traffic. This leads to increased packet delay, loss of packets, less accuracy. Transmission speed is a factor that contributes to the operation of WSN. We presented a mechanism for adjusting transmission speed to solve this problem. In this work, biodiversity-based monitoring system called BatFuzzyBee is presented. It defines WSN bio definitions that use location information to manage congestion. New cluster-based WSNs are also used in this work to reduce packet loss and save energy. BatFuzzyBee can find the best or closest reliable route, when the network has nodes with different transmission ranges with the least power consumption. The proposed BatFuzzyBee is compared to other methods by considering multiple indicators, such as packet delivery ratio, accuracy and energy consumption. The results from the simulation show that the proposed method gives better performance when compared with the other methods. In this way, the effectiveness of the BatFuzzyBee approach is determined.
\end{abstract}

Keywords: Congestion Control; Wireless Sensor Networks (WSNs); Bat Optimization ; Bee Optimization; BatFuzzyBee

\section{Introduction}

A large number of distributed device nodes make up a wireless sensor network (WSN). The sensor unit's data is transferred to the base station. Sensors can monitor animals, detect floods, forecast meteorological data, track patients and track vehicles. Sensor nodes perform independent monitoring tasks and perform certain processes. The monitored data is collected by the relay device and directed to the base station. Sensors are capable of operating in difficult situations. However, the sensor unit has limited battery life, low memory and fast deceleration.

Some sensors that are spread across a large geographic area gives greater rate of transmission. Overloading can result in packet loss and response times being sped up. Reduced network traffic causes response time to be delayed in busy networks. Dealing with congestion in the WSN necessitates special thought. Adjusting the baud rate of each node solves the congestion problem. Several transmission speed control techniques have been presented in recent years. To avoid transmitting and losing packets, the frequency of incoming and outgoing data packets must be enabled. To avoid packet loss, overloading should be monitored through each network. The use of traffic congestion avoidance methods can be beneficial.

Previous research on data transfer and quality of service assurance has been split into numerous categories:

(i) approaches for reducing the amount of data generated by the device node (ii) techniques for detecting and controlling congestion based on the node's queue state (iii) Techniques for detecting congestion using package service time (iv) Techniques for correcting receiving node crossing points by passing congestion to surrounding nodes and (v) Techniques for employing packet priority. All of these methods have the drawback of necessitating more transmission. To overcome these problems, an optimal route detection approach is needed. So this work introduced BatFuzzyBee approach. The major contribution of this work is to resolve and reduce congestion in the WSN using the BatFuzzyBee optimization method. Another major contribution is the implementation of congestion control techniques presented in the WSN and the results are analysed and compared with existing methods to prove the efficacy of the proposed method. 


\section{Related Work}

Authors of [5] solves the WSN congestion problem by modifying the transmission rate in the current node. A support vector machine does multifunctional diversification (SVM). In [25] GWO provides greater performance with less computing time and a higher success rate. With GPS devices, known nodes are referred to as auxiliary nodes. Anchors use GPS devices to determine their location. To locate nodes, it can be integrated with other algorithms. When nodes are used in an unfavorable context, data collecting becomes unreliable. Mechanisms for detecting abnormalities have been proposed previously in order to gather reliable information. It is quite useful to detect anomalies in the sensor network in order to make a choice based on the data obtained.

At WSN, the author [11] requested a protocol for cluster transmission. A three-tiered hybrid algorithm was utilized to improve network performance. Nature-based approaches are used in the Multilevel Hybrid Protocol (MLHP). Cluster heads are chosen at the start and data transport is handled via grey wolf optimization. With the help of GWO, the node chooses the optimum approach to save energy. Group distribution is used at the multilevel. In WSN, SHPP delivers more residual power, greater network stability and longer network life. The location of an unknown node is a critical issue to resolve.

Authors in [15] are a group of researchers who have worked on a number of different projects to limit the length of the cache queue, proposed overload avoidance strategies based on the initial detection algorithm RED), which is extensively used in router queue management. It was used in a wireless sensor network and used to manage congestion at the basic level.

The authors of [4] were the first to raise the issue of single classification. One Class SVM solves the challenge of finding anomalies (OCSVM). The reference vector engine is shown to be a useful classification tool. In an ODM, the main function can be utilized. The ODCMM is used to perform optimization with the goal of detecting abnormalities. The authors of [6] took great care to find errors by using SVM to classify sensor data. The Online Sparse Least Squares SVM was used to detect sensor error(OS-LSSVM). The Error Correcting Code SVM was used to extract the error feature (ECOC-SVM). The first character is separated and the faulty state is classified. To reduce sensor node localization errors, several optimization strategies are applied.

To solve this optimization challenge, the authors of [21] utilised meteorology. To predict the position of device nodes, Particle Swarm Optimization (PSO) algorithms and Wolf Algorithm are utilised. Localization issues are solved by using optimization algorithms to reduce localization errors. GWO outperforms all other solutions in terms of efficiency and error reduction. Calculating this, however, takes a long time. Better network life is a difficult with WSN since sensors have less power and run out of power faster. GWO is better than other optimization techniques, according to [8]. It provides improved accuracy and most crucially, allows GWO to operate in a low-power environment in less time.

To solve data compression and weighing concerns in existing WSN congestion algorithms, Authors of [12] proposed a new congestion management algorithm using electronic estimation and weighing. In [13] authors suggested a new approach of congestion management that estimates congestion levels by combining buffer range lengths and associated characteristics. It distinguishes between the states of the nodes and uses different bandwidth allocation algorithms for each state, ensuring reliable data transfer. In reaction to congestion happening before the overflow queue, communication network equipment implements Active Queue Management (AQM) [14].

We can improve WSN transmission at the corresponding parameter values by comparing different experimental outcomes at different experimental parameters. The present congestion control technique is based on technology that designs network controllers capable of addressing the control requirements of WSN data streams using automated control theory. The WSN was upgraded using Proportional Integration control technology and the bandwidth for repository data in the network was designated as a controlled object [9]. The purpose of PI controllers is to accomplish active range control. [10] proposes an active tail control system that combines PI control techniques with algorithms to improve titanium particle excitability. The first step is to create a better IP controller model. The bubble size of the quantum particles was then optimized using an algorithm to optimize the parameters in the IP controller model.

The proposed method achieves good congestion control with WSN with low packet loss rate and high network bandwidth, according to experimental results. Algorithms for regulating quantum particle congestion that take equity into consideration have also been presented. To adapt to complicated WSN environments, quantum particle swarm optimization (QPSO) was first incorporated into a proportional integration algorithm. The likelihood of the range between the nodes is then changed with varied transmission distances from the sink to increase the accuracy of the network load [18] based on network congestion and packet transmission distance. 


\section{Materials and Methods}

Congestion can occur in the WSN while collecting information and sending it to the sink. This leads to increased packet delay, packet loss, severe deterioration of accuracy and power loss of nodes. The main aim of the proposed work is to eliminate congestion by using optimal route identification schemes in which each node transmits data in the best possible way, thus ensuring fairness as a priority and minimizing packet loss. The proposed congestion control technique will help to optimize the whole system by supporting the delivery of critical events to the sink. To find the optimal path, a BatFuzzyBee approach is introduced. The main function of BatFuzzyBee is shown in Fig. 1. For detection of congestion in WSN, the proposed approach uses three main function.

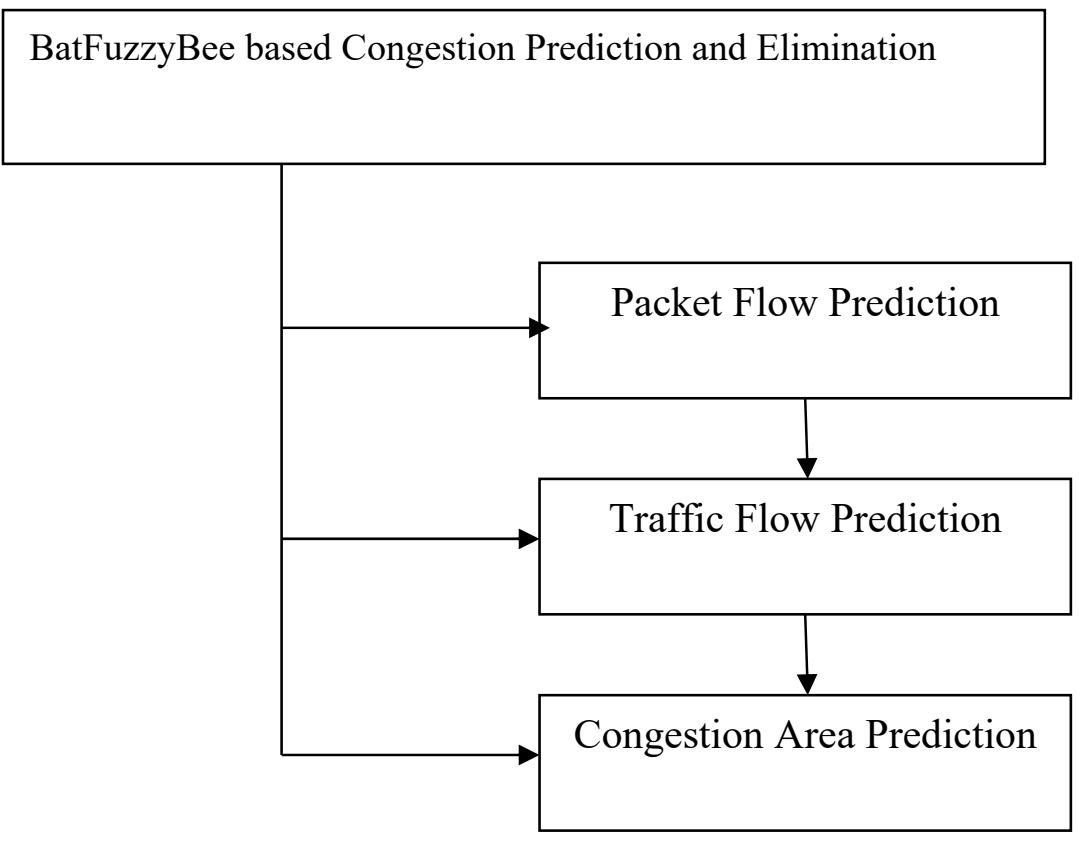

Fig 1: Proposed Congestion Prediction functions

\subsection{Proposed Methodology}

This approach has three phases. The first phase is to create network model. In this phase, network is created with nodes. All nodes have some properties like private id, public id, neighbours, initial energy. After completing this, a congestion model is created with the help of nodes and routing protocols. The final phase is to predict and eliminate congestion in WSN using proposed BatFuzzyBee. The general architecture of Proposed Congestion Control Approach is shown in Fig. 2.

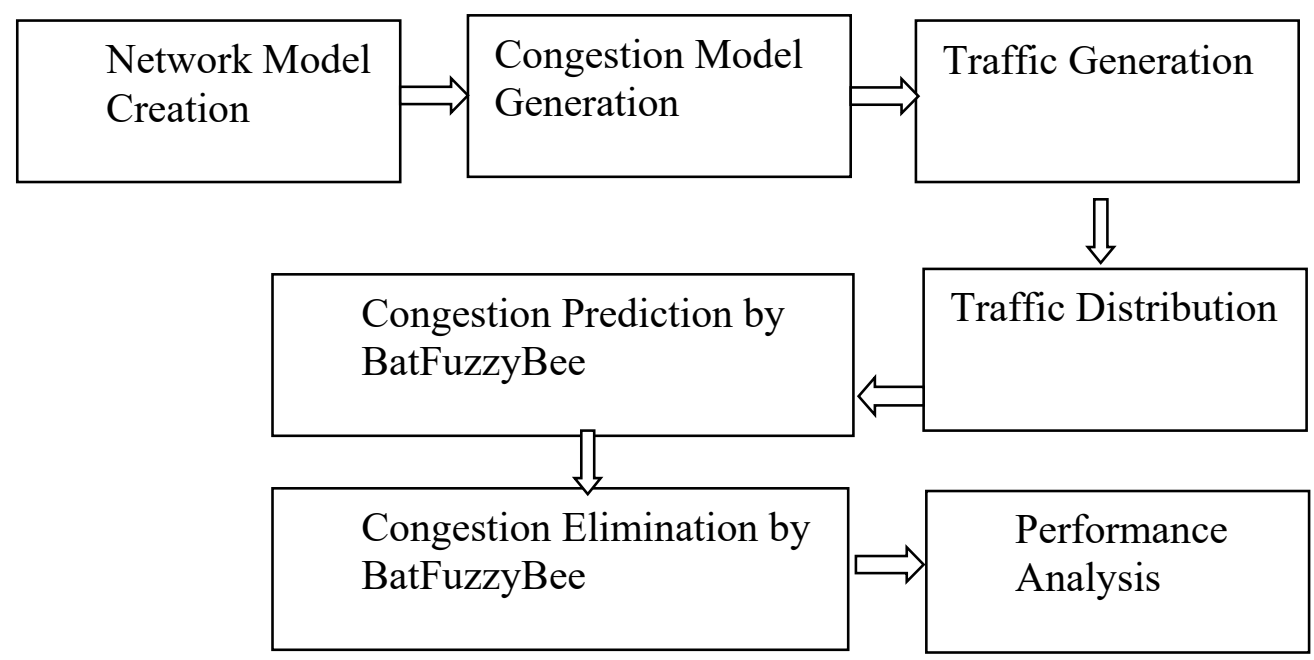

Fig 2: The General Architecture of Presented Approach 
Major steps of presented approach are

1. Network Model Creation

2. Congestion Model Generation

3. Congestion Prediction and Elimination using BatFuzzyBee

\subsubsection{Network Model Creation}

The model of the presented work has four regions. Each region contains a number of nodes. Among these nodes one region head is selected based on weight. The rest of all nodes in each region is called Region Member(RM). The members can send data to their Region $\operatorname{Head}(\mathrm{RH})$. All region heads are interconnected. So they can communicate with each other. To collect the data from all region, mobile sinks are used. In this work, these mobile sinks are called batbees. It is shown in Fig. 3.

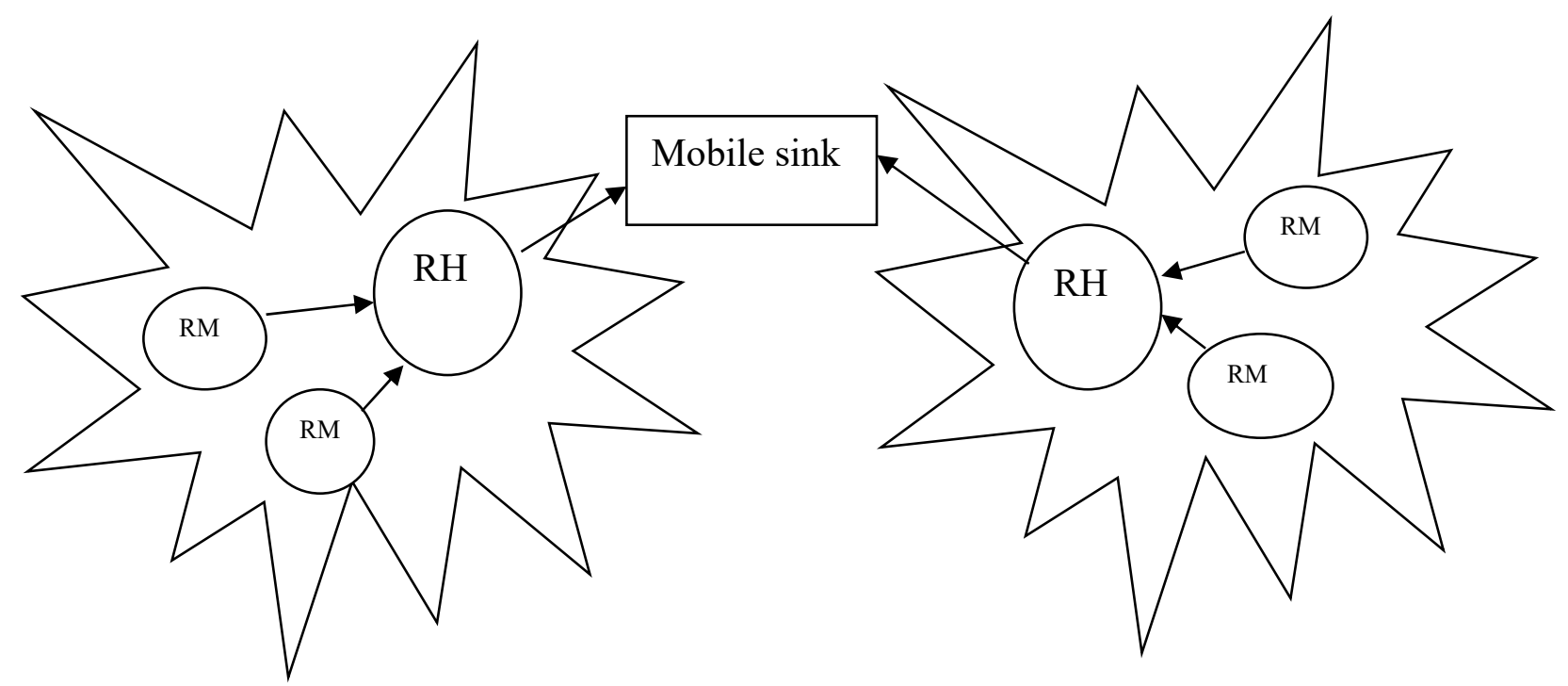

Fig 3: Network Model creation of Proposed Work

\subsubsection{Congestion Model Generation}

Congestion is a state of traffic congestion and consequently it affects the quality of service in the network. The major reason for WSN congestion is due to the load imbalance where the device node load outdo the available buffer capacity. An ideal environment for congestion needs to be created for the purpose of predicting congestion. So this work creates a pattern of congestion by following the two steps.
a) Traffic Generation
b) Traffic Distribution

\section{a) Traffic Generation}

This uses a region based WSN. Node aggregation forms regions. In a given region, multiple nodes will act as $\mathrm{RM}$, which is responsible for collecting environmental data. A node will act as RH. All RM transmits the collected data to RH. The RH in turn connects to a storage device. Each region has RHs. When the transmission rate of RM exceeds the receiving capacity of RM, the data transmitted by $\mathrm{RH}$ must be in range. Therefore, the $\mathrm{RM}$ queue size is also an important factor. If the size of the queue is not enough, traffic congestion occurs. 


\section{b) Traffic Distribution}

In WSN, when the number of packets arrives, increasing the network capacity or node, congestion occurs in the network. In this work, the DSR protocol is used to create traffic segments. It is a representative of the dynamic protocol for remote vector transmission. DSR is a reaction routing protocol. It means that the path is not created until it is required by the node that will send the data to the destination. Nodes do not store information about each other until the first packet is requested to be sent to the destination. The Fig.4 is showing the traffic distribution scheme.

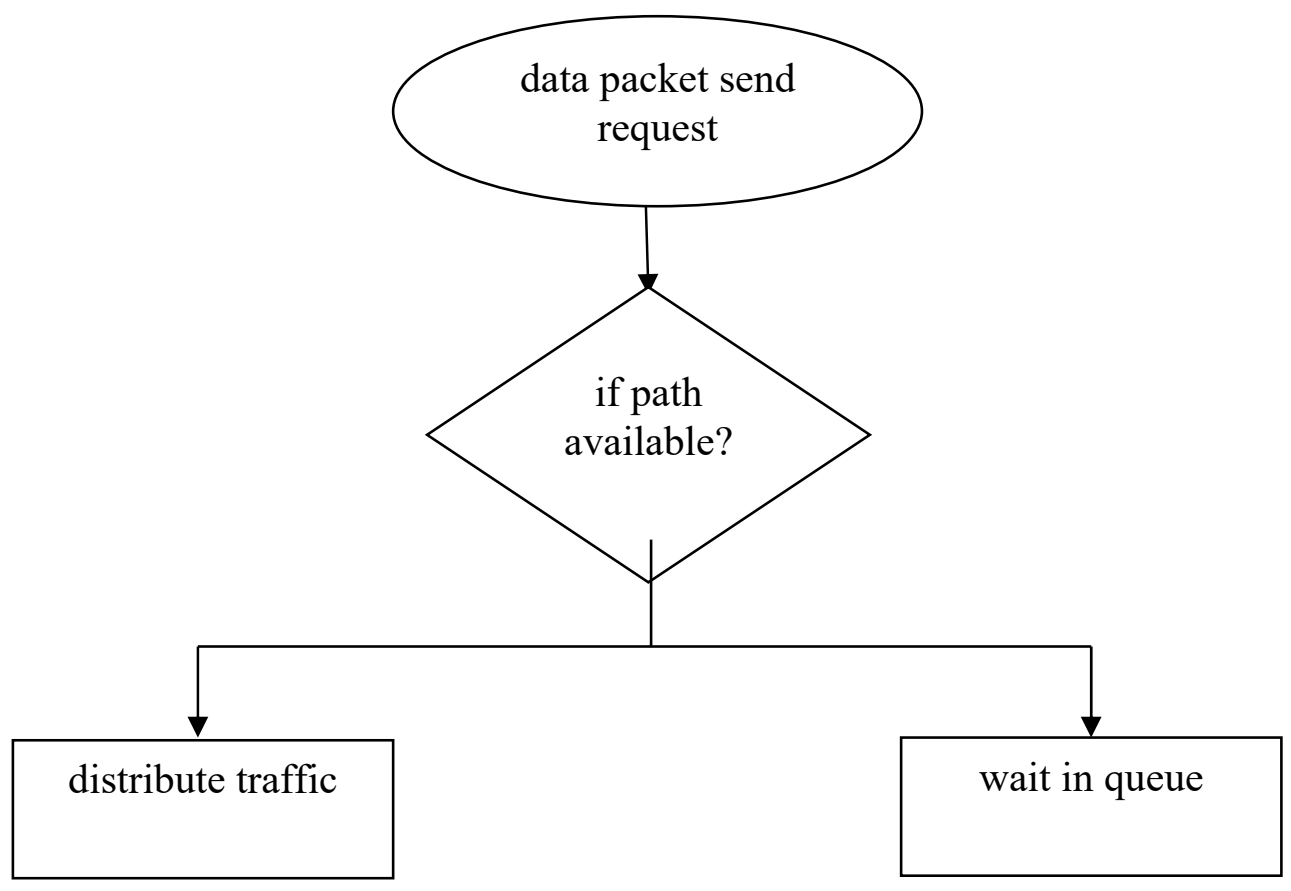

Fig 4: Traffic distribution

\subsubsection{Congestion Prediction and Elimination by BatFuzzyBee}

In this work, BatFuzzyBee approach is used for prediction and controlling congestion. This is a combination of bat, bee optimization algorithms. The Bat optimization was incorporated with bee for better design, which improves the performance of the algorithm .

The simulated BatFuzzyBee algorithm is described as follows:

\footnotetext{
Algorithm : BatFuzzyBee

Step 1: Start creating parameters of the optimization algorithm. Each head of the cluster is considered a batbee. Step 2: Determine the suitability of heads of all cluster and the most suitable is determined as the best. Step 3: The cluster head is optimally scaled by adjusting the frequency, speed and position.

Step 4: The cluster head moves around when a random number is generated.

Step 5: First a random number is selected to select the new cluster head, and when the random number is greater than the quantity and suitability of the newly selected cluster head, it proves to have better efficacy than the previous cluster head.

Step 6: Batbee ranks and determines the best currently.

Step 7: The above steps are repeated until the stop criteria are reached.
}

Further for congestion find the fitness value and compare with congestion control rate. If the congestion is there, then congestion prediction flag value is 1 , otherwise CPF is 0. BatfuzzyBee provides best efficient solution for congestion problem. 


\section{Experimental Results}

The presented system is implemented through NS2. Performance metrics like Packet Delivery Ratio (PDR), Average Throughput (AT), Detection Accuracy, Energy consumption, loss of packet, network lifetime are analysed and results are presented. To demonstrate this, a scenario is applied where the value of the proposed BatFuzzyBee based congestion management strategy is compared with other congestion control approaches by altering the number of nodes upto 50. Hence it is justified that performance of the of BatFuzzyBee is better than Lion, Bee, and LionFuzzyBee methods in controlling congestion in WSN.

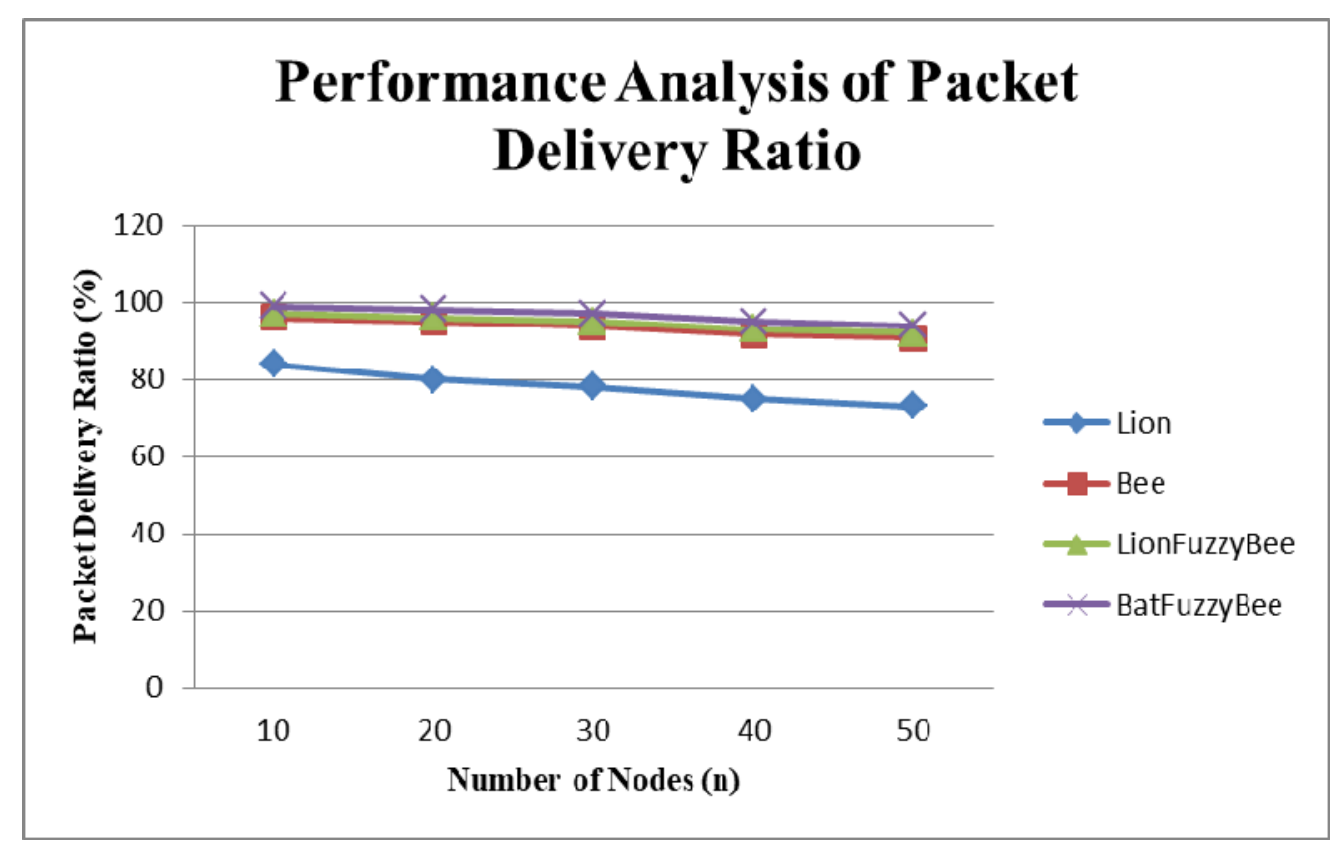

Fig 5: Evaluation Output of Packet Delivery Ratio

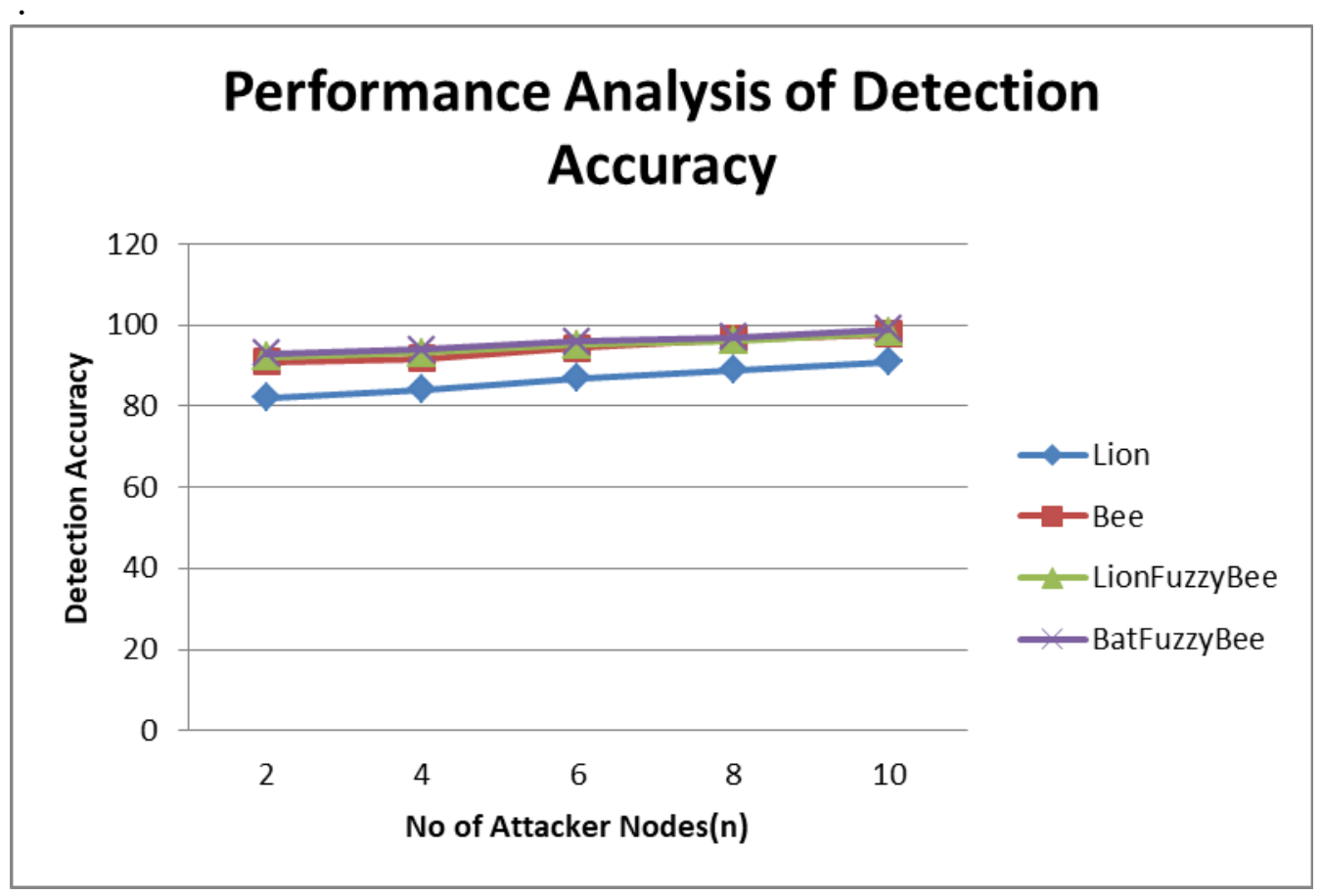

Fig 6: Evaluation Output of Detection Accuracy 


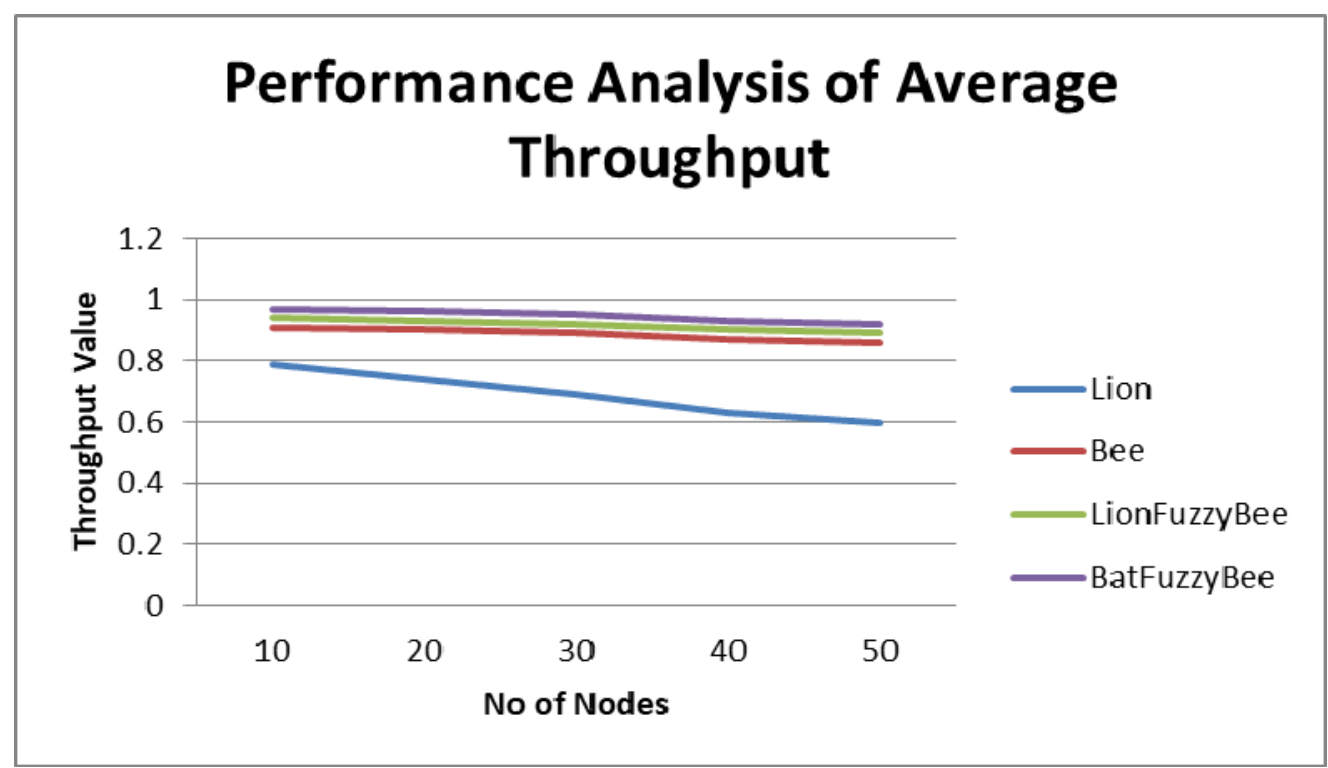

Fig 7: Evaluation Output of Average Throughput

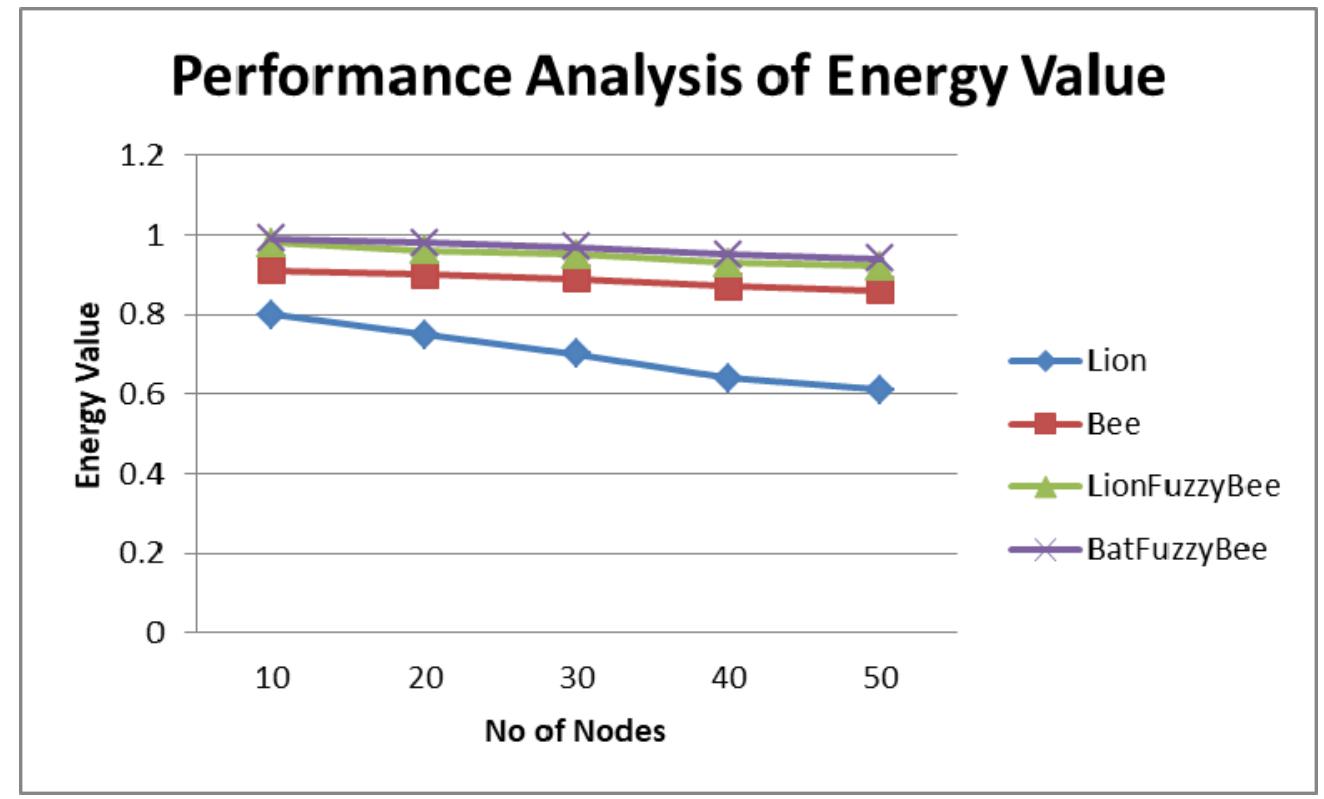

Fig 8: Evaluation Output of Energy Value 


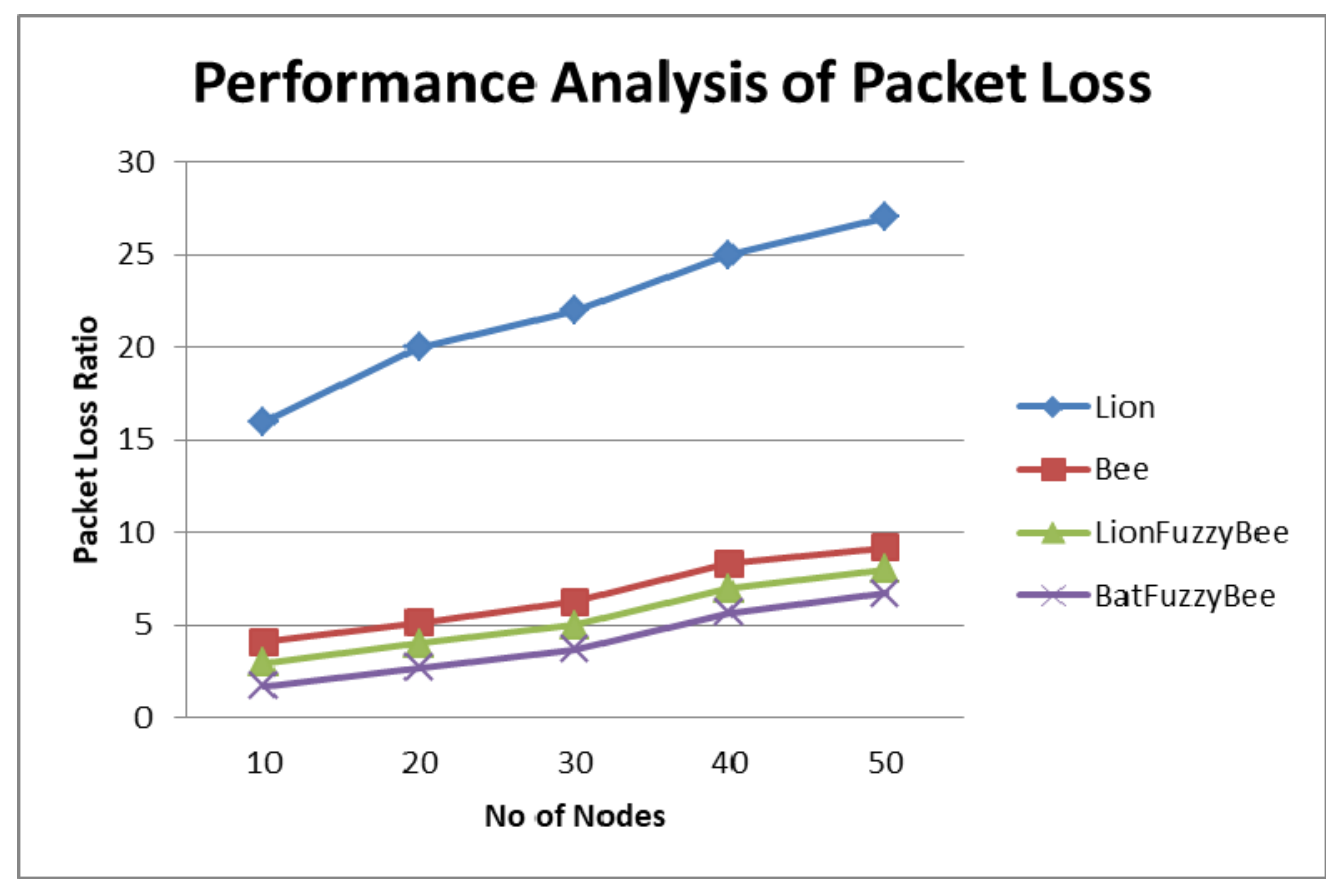

Fig 9: Evaluation Output of Packet Loss

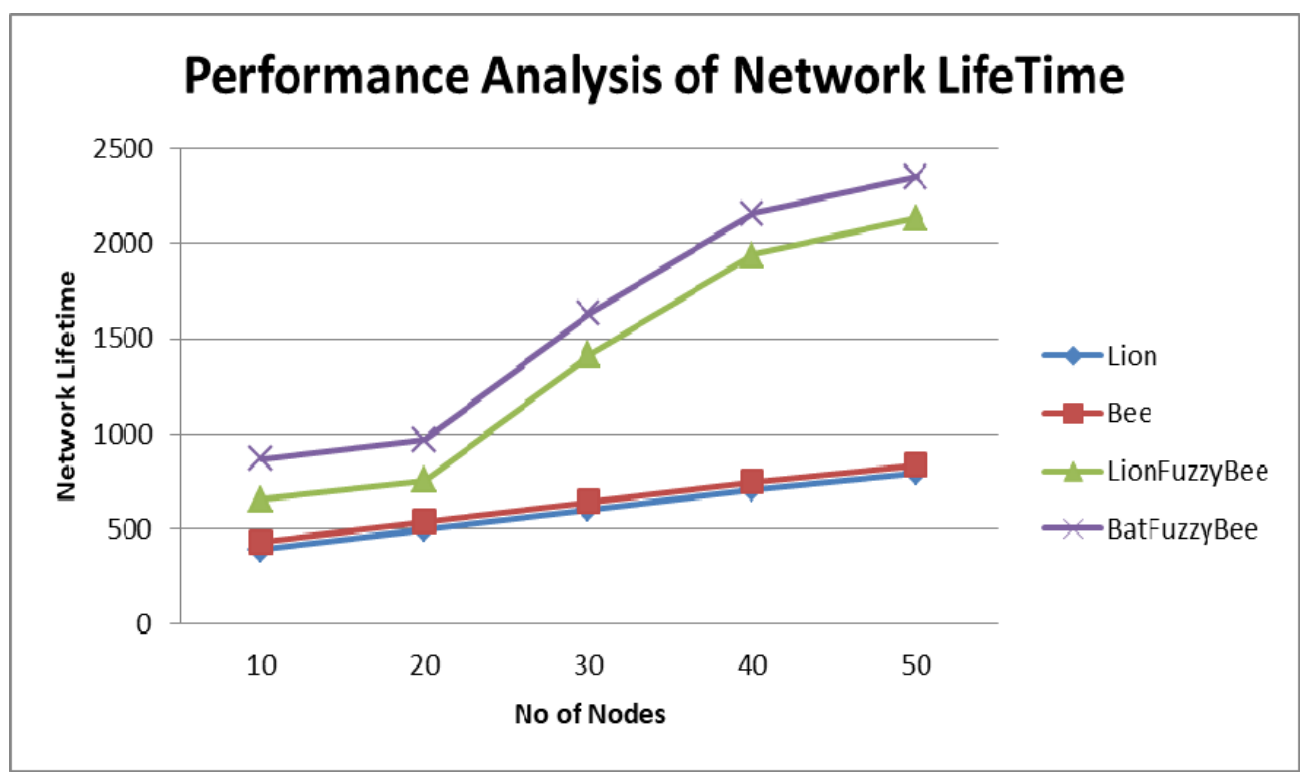

Fig 10: Evaluation Output of Network Lifetime

\section{Conclusion}

WSN congestion management is a challenging area. Resource constraints make the task of developing congestion management techniques more difficult and complex. This article has proposed a BatFuzzyBee method. BatFuzzyBee techniques are classified as central and distributed strategies based on primary and secondary design objectives. This technique is thoroughly discussed and evaluated using the various performance and design indicators used to measure congestion. The experimental output has justified that congestion areas are predicted with more accuracy using BatFuzzyBee. Any change in network utility need to be analysed which is dependent on how congestion area is designed . BatFuzzyBee has achieved highest network life time due to reduction of energy loss and packet loss through elimination of congestion. 


\section{References}

[1] Garcia, Miguel, et al. "Saving energy and improving communications using cooperative group-based wireless sensor networks," Telecommunication Systems, 52(4), pp: 2489-2502, 2013.

[2] Lee, J.H.; Jung, I.B. Adaptive-compression based congestion control technique for wireless sensor networks. Sensors 2010, 10, 29192945.

[3] Dervis Karaboga, “Artificial Bee Colony Algorithm”, 2010, Scholarpedia, 5(3):6915.

[4] Tran, K.P. and Huong, T.T., 2017, October. Data driven hyperparameter optimization of one-class support vector machines for anomaly detection in wireless sensor networks. In Advanced Technologies for Communications (ATC), 2017 International Conference on (pp. 6-10). IEEE.

[5] Gholipour, M., Haghighat, A. T., \& Meybodi, M. R.. Hop-by- Hop Congestion Avoidance in wireless sensor networks based on genetic support vector machine. Neurocomp, 223, 63-76. 2017.

[6] Deng, F., Guo, S., Zhou, R. and Chen, J., 2017. Sensor multifault diagnosis with improved support vector machines. IEEE Transactions on Automation Science and Engineering, 14(2), pp.1053-1063.

[7] Kemal Akkaya, Mohamed Younis "A survey on routing protocols for wireless sensor networks", IEEE Comm Magazine on AdHoc Networks, pp.325-349, 2005

[8] Shieh, C.S., Sai, V.O., Lee, T.F., Le, Q.D. and Lin, Y.C., 2017. Node Localization in WSN using Heuristic Optimization Approaches, International Conference on Networking and Network Applications, 2016

[9] Yin, Y.F.; Yuan, H.L.; Zhang, B.L. Dynamic behavioral assessment model based on Hebb learning rule. Neural Computing and Applications, 2017, 28, 245-257.

[10] Sun, P.L.; Niu, L.G. Adaptive congestion control algorithm based on predictive control. In Proceedings of the IEEE Conference Publications, 2008, pp. 644-648.

[11] Al-Aboody, N.A. and Al-Raweshidy, H.S., 2016, September. Grey wolf optimization-based energy-efficient routing protocol for heterogeneous wireless sensor networks. In Computational and Business Intelligence (ISCBI), 2016 4th International Symposium on (pp. 101-107). IEEE.

[12] Li, G.H.; Li, J.Z.; Gao, H. ع-Approximation and Weighted Fairness Guaranteed Congestion Control Algorithm for Wireless Sensor Networks. 2011, 34, 2197-2210.

[13] Sun Hua, Congestion Control Based on Reliable Transmission in Wireless Sensor Networks. Journal of Networks, $2014,9,762-768$.

[14] Gu, D.; Zhang, W. Design of an Hळ based PI controller for AQM routers supporting TCP flows. In Proceedings of the 48th IEEE Conference, 15-18 December 2009; pp. 603-608.

[15] Li, L.W.; Yang, H.Y. Congestion Control Strategy Based on RED Algorithm in Wireless Sensor Network. Comput. Simul. 2012, 3, $13-16$.

[16] Zhao, S.J.; Wang, P.P.; He, J.H. Simulation Analysis of Congestion Control in WSN Based on AQM. In Proceedings of the 2011 International Conference on Mechatronic Science, Electric Engineering and Computer (MEC), 19-22 August 2011; pp. 197-200.

[17] X. Wang and K. Kar, "Distributed algorithms for max min fair rate allocation," in ALOHA networks, Proc Allert Conf, 2004.

[18] Li, X.L.; Chu, Z.G. Design of Wireless Sensor Network Congestion Control Algorithm Based on Active PI Model and Improved Quantum Particle Swarm Optimizing Algorithm. Comput. Meas. Control 2014, 22, 3656-3658.

[19] Liqiang Tao, Fengqi Yu , "ECODA: enhanced congestion detection and avoidance for multiple class of traffic in sensor networks." IEEE transactions on consumer electronics, 56.32010.

[20] C. Caione, D. Brunelli, and L. Benini, "Distributed compressive sampling for lifetime optimization in dense wireless sensor networks," IEEE Transactions on Industrial Informatics, vol. 8, no. 1,2012.

[21] Kaur, R. and Arora, S., 2017. Nature inspired range based wireless sensor node localization algorithms. International Journal of Interactive Multimedia and Artificial Intelligence, 4(6), pp.7-17.

[22] Wan, Chieh-Yih, Shane B. Eisenman, and Andrew T. Campbell. "CODA: Congestion detection and avoidance in sensor networks." Proceedings of the 1st international conference on Embedded networked sensor systems, ACM, 2003.

[23] Zhu, L., Zhang, Z., \& Xu, C. Secure data aggregation in wireless sensor networks. In Secure and Privacy-Preserving Data Communication in Internet of Things(pp. 3-31). Springer Singapore,2017.

[24] Chen, H. M., Cui, L., \& Zhou, G. (2017). A Light-Weight Opportunistic Forwarding Protocol with Optimized Preamble Length for Low-DutyCycle Wireless Sensor Networks. Jour of Comp Science and Tech, 32(1), 168-180.

[25] Rajakumar, R., Amudhavel, J., Dhavachelvan, P. and Vengattaraman, T., 2017. GWO-LPWSN: Grey wolf optimization algorithm for node localization problem in wireless sensor networks. Journal of Computer Networks and Communications, 2017

[26] Selim Yilmaz and Ecir U. Kucuksille, "Improved bat algorithm (IBA) on continuous optimization problems," Lecture Notes on Software Engineering, 2013,1(3), pp: 279-283.

[27] Kang, Jaewon, Yanyong Zhang, and Badri Nath. "TARA: topology-aware resource adaptation to alleviate congestion in sensor networks." IEEE Transactions on Parallel and Distributed Systems ,18.7, 919-931, 2007 